\title{
Preoperative hemogram-related parameters to distinguish renal cell carcinoma from benign kidney masses: HERR score
}

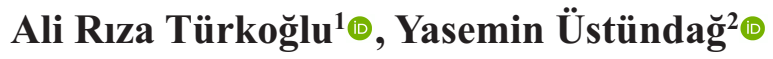 \\ ${ }^{1}$ Department of Urology, University of Health Sciences, Bursa Yüksek Ihtisas Training and Research Hospital, Bursa, Turkey \\ ${ }^{2}$ Department of Clinical Biochemistry, University of Health Sciences, Bursa Yüksek Ihtisas Training and Research Hospital, Bursa, \\ Turkey
}

DOI: $10.18621 /$ eurj.408088

\begin{abstract}
Objectives: Renal cell carcinoma (RCC) accounts for approximately $90 \%$ of all kidney malignancies, and it is difficult to preoperatively distinguish between tumors and benign masses without a kidney biopsy in small renal masses. We investigated whether any preoperatively defined hemogram-related parameters had a predictive value that would distinguish RCC from benign kidney masses using a novel scoring method.

Methods: Between January 2011 and November 2017, 330 patients diagnosed with kidney masses and who received an operation were included. Fifty-six masses were benign. The neutrophil-to-lymphocyte count (NLR), platelet-to-lymphocyte count, lymphocyte-to-monocyte count, mean platelet volume, platelet count ratio, and hemoglobin to red cell distribution width ratios were calculated. The hemogram-related parameters were combined with the tumor size to establish the hemogram-related risk (HERR) score. The area under the receiver operating characteristics curve, sensitivity, specificity, and likelihood ratios were evaluated to preoperatively diagnose RCC.

Results: Histological findings confirmed RCC in 274 patients. The NLR [median (interquartile range)] was higher in patients with RCC, 3.7 (4.7), compared to a benign kidney mass, $2.4(2.2)(p<0.001)$. A HERR score cut-off of $\geq 3$ showed a good sensitivity at $78 \%$ with an LR + of 10.8 [95\% confidence interval (CI): 7.0-16.4] and an LR- of 1.2 (95\% CI: 1.0-1.5).

Conclusion: Our study, despite being a preliminary validation, is the first to evaluate hemogram-related parameters for preoperatively discriminating between RCC and benign renal masses, and the HERR score serves as a potential diagnostic biomarker for this
\end{abstract}

Keywords: Renal cell carcinoma, kidney mass, hemogram-related risk score

Received: March 20, 2018; Accepted: March 28, 2018; Published Online: April 7, 2018

$\mathbf{R}$ enal cell carcinoma (RCC) is the most common solid kidney lesion, and it accounts for approximately $90 \%$ of all kidney malignancies [1] Old age, hypertension, and lifestyle factors, such as smoking and obesity, are among the etiologic factors of $\operatorname{RCC}[1,2]$.
Kidney masses are mostly asymptomatic until the late stages of the disease. However, with the development and widespread use of imaging modalities, such as ultrasound and computed tomography, the incidental diagnoses of RCC are increasing [3]. Because of the earlier diagnosis, these

Address for correspondence: Ali Rıza Türkoğlu, MD., University of Health Sciences, Bursa Yüksek Ihtisas Training and Research Hospital, Department of Urology, Bursa, Turkey

E-mail: a.turkoglu@hotmail.com 
tumors are usually smaller in size and low-grade [4]. Studies indicate that it's difficult to distinguish between benign and malign kidney tumors radiologically without a kidney biopsy in small renal masses [5]. In fact, multiple studies demonstrated that the incidence of surgically treated benign renal tumors can be up to $20 \%[4,6]$.

Host inflammatory response plays critical role in the initiation and progression of various malignancies, including RCC [7]. Cancer patients frequently present with systemic inflammatory responses as alterations in peripheral blood cell counts. Recently, a number of studies about circulating blood-cell-based biomarkers, such as the neutrophil-to-lymphocyte ratio (NLR) and platelet-to-lymphocyte ratio (PLR), which reflect the systemic inflammation, were shown to be effective predictors of the prognosis across various cancers, including RCC $[8,9]$.

A low lymphocyte-to-monocyte count ratio (LMR) was shown to be indicative of the aggressiveness of RCC [9]. Meanwhile, the hemoglobin $(\mathrm{Hb})$ to red cell distribution width (RDW) ratio has been shown to be a new prognostic parameter in cancer patients [10]. A low preoperative MPV and high platelet count were demonstrated to be independent predictors of tumor-specific mortality in RCC patients [11]. The identification of noninvasive markers that can be used to screen RCC patients in the early stages is one of the challenges of urologists [12]. Currently, there is no diagnostic biomarker available for an accurate diagnosis of RCC other than incidental radiological discovery.

In this study, we aimed to investigate whether any of the preoperatively defined hemogram-related parameters, namely NLR, PLR, Hb to RDW, MPV to platelet count, and lymphocyte-to-monocyte count, had a predictive value for distinguishing RCC from benign kidney masses and to design a scoring system for this purpose.

\section{METHODS}

This study was performed in accordance with Declaration of Helsinki in 1965 (as revised in 2013). Ethical clearance was granted by the Regional Ethics Committee. We performed a search of the database of our hospitals' Laboratory Information System, which integrates information from several databases and includes the patient demographics, clinical diagnosis, order entry database, and laboratory results database. Between January 2011 and November 2017, patients diagnosed with a kidney mass and who had a radical nephrectomy or partial nephrectomy treated by either open or laparoscopic methods in our institute were included in the study. The exclusion criteria included patients with other known malignancies, hematologic diseases, autoimmune diseases, active infections, preoperative blood transfusions, those under anticoagulant treatment or prior steroid or anticancer therapy, or patients where perioperative routine laboratory tests were unavailable.

A total of 354 patients with a complete blood panel performed within 30 days prior to the surgery and available medical records were discovered. Finally, 330 out of 354 patients were included in the study, and 56 of them had benign tumors. The longest diameter of the tumor size (TS), histological cancer type, T-stage, Fuhrman grade, status of lymph node metastasis, and necrosis, defined as the presence of microscopic coagulative necrosis, were taken from the pathology reports.

The NLR was calculated by dividing the absolute number of neutrophils by the lymphocyte count. The PLR was calculated by dividing the absolute platelet count by the lymphocyte count. MPV to platelet count ratio, $\mathrm{Hb}$ to RDW ratio, and lymphocyte-to-monocyte count ratios were also calculated.

\section{Statistical Analysis}

Statistical analysis was performed using the Statistical Package for the Social Sciences (SPSS, version 21; SPSS Inc., Chicago, IL, USA). The normality of the continuous variables was analyzed with the Kolmogorov Smirnov test. Results are expressed as mean \pm standard deviation (SD) or median (interquartile range). Normally distributed continuous variables were compared using the independent-samples t-test, and the Mann-Whitney U test was used if the distribution was skewed. Categorical data were compared using the chi-square test. The Kruskal-Wallis test was used for computing differences across groups. A $p$-value of 0.05 was considered to be statistically significant.

The ROC curve, a parameter that reflects the sensitivity and specificity of continuous variables, was 
used to determine the cut-off values of the parameters. Youden's index was applied to determine the optimal cut-off value.

Next, we stratified the hemogram-associated markers and analyzed them as categorical variables. We combined the hemogram-related parameters with tumor size to establish the hemogram-related risk (HERR) score as follows: patients with increased NLR ( $\geq 2.47$ and $<4.0,1$ point; $>4.0,2$ points), and MPV to $\operatorname{PLT}(\geq 0.028,1$ point), tumor size ( $\geq 4 \mathrm{~cm}, 1$ point), decreased LMR ( $\leq 4.20,1$ point), and $\mathrm{Hb}$ to RDW ratio $(\leq 0.86,1$ point). The HERR score was calculated by totaling these individual scores. Using the postoperative pathological report as the gold standard, the sensitivity (SE), specificity (SP), positive and negative predictive values (PPV, NPV), positive likelihood ratio $(\mathrm{LR}+)$ and negative likelihood ratio (LR-) were calculated.

\section{RESULTS}

The histological findings confirmed RCC in 274
Table 1. The diagnosis of patients

\begin{tabular}{lc}
\hline Diagnosis & Number of patients \\
\hline Benign masses & $\mathbf{5 6}$ \\
Renal cyst & 26 \\
Renal cortical adenoma & 2 \\
Oncocytoma & 15 \\
Anjomyolipom & 13 \\
Renal cell cancers & $\mathbf{2 7 4}$ \\
Clear renal cell carcinoma & 225 \\
Papillary renal cell carcinoma & 26 \\
Chromophobe renal cell carcinoma & 11 \\
Unclassified renal cell carcinoma & 12 \\
\hline
\end{tabular}

patients (Table 1). Of these, 168 (61\%) were men, and $106(39 \%)$ were women. The mean age of the patients with benign masses was $58.5 \pm 12.7$ ( 22 to 82 years) and $59.5 \pm 11.9$ (18 to 89 years) for malignant cases (Table 2). Out of these total RCC patients, 225 (82\%) had renal clear-cell carcinoma, $26(9.4 \%)$ had a papillary RCC, 11 (4\%) had a chromophobe RCC, and $12(4 \%)$ were unclassified.

In the benign group, 17 of the patients had a tumor

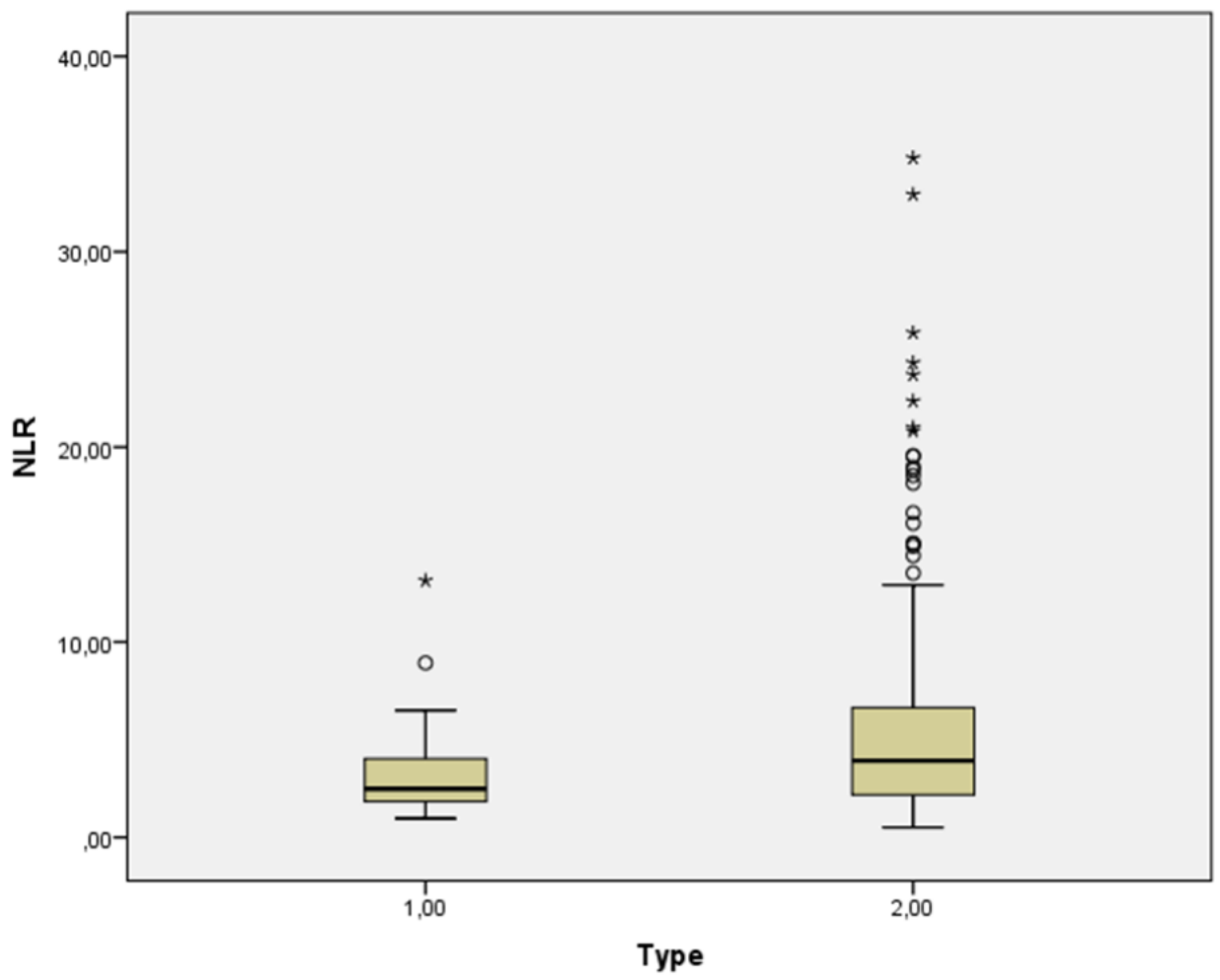

Figure 1. Neutrophil to lymphocyte ratio. The bar graphs show blood neutrophile to lymphocyte (NLR) levels in benign (type 1) and renal cell carcinoma (type 2) patients. 
size $\leq 4 \mathrm{~cm}, 64$ of the RCC patients had a tumor size $\leq 4 \mathrm{~cm}$. Three of the benign patients had a tumor size $>10 \mathrm{~cm}$, and 53 of the RCC patients had a tumor size $>10 \mathrm{~cm}$. When the patients were stratified according to pathological stage, $51 \%$ were at stage pT1, 24\% at stage pT2, $21 \%$ at stage pT3, and $2 \%$ at stage pT4 (Table 2). When renal clear-cell patients were stratified according to the Fuhrman grade, 47 patients were classified as grade I, 120 as grade II, 45 as grade III, and 14 as grade IV. Of the RCC patients, 154 underwent an open radical nephrectomy, 76 had a laparoscopic radical nephrectomy, 38 had an open partial nephrectomy, and 6 had a laparoscopic partial nephrectomy. In the benign patient group, 22 underwent an open radical nephrectomy, 16 had a laparoscopic radical nephrectomy, 14 had an open partial nephrectomy, and 4 had a laparoscopic partial nephrectomy.

The NLR levels [median (interquartile range)] were higher in the patients in the malignant kidney mass group with a value of 3.7 (4.7) compared to a value of 2.4 (3.2) for the benign kidney mass group $(p$ $<0.001$; Figure 1). The RDW, PLT, LMR, PLR, Hb to RDW, and MPV to PLT levels were similar between

Table 2. Characteristics of the entire study population $(n=330)$

\begin{tabular}{|c|c|c|c|}
\hline & $\begin{array}{c}\text { Benign } \\
(n=56)\end{array}$ & $\begin{array}{c}\text { RCC } \\
(n=274)\end{array}$ & $p$ value \\
\hline Gender (M/F) & $31 / 25$ & $170 / 104$ & 0.018 \\
\hline Age (years) & $58.5 \pm 12.7$ & $59.5 \pm 11.9$ & 0.995 \\
\hline \multicolumn{4}{|l|}{ TNM Stage } \\
\hline pT1a & & 60 & \\
\hline $\mathrm{pT} 1 \mathrm{~b}$ & & 81 & \\
\hline $\mathrm{pT} 2 \mathrm{a}$ & & 34 & \\
\hline $\mathrm{pT} 2 \mathrm{~b}$ & & 34 & \\
\hline pT3a & & 49 & \\
\hline $\mathrm{pT} 3 \mathrm{~b}$ & & 7 & \\
\hline pT3c & & 3 & \\
\hline pT4 & & 6 & \\
\hline WBC $\left(10^{9} / \mathrm{L}\right)$ & $7.9(3.7)$ & $9.2(5.0)$ & $0.001 *$ \\
\hline Neutrophile $\left(10^{9} / \mathrm{L}\right)$ & $5.0(2.5)$ & $6.3(4.7)$ & $<0.001 *$ \\
\hline Lymphocyte $\left(10^{9} / \mathrm{L}\right)$ & $1.8(1.0)$ & $1.7(1.1)$ & 0.113 \\
\hline RDW & $14.2(2.1)$ & $14.5(2.3)$ & 0.843 \\
\hline $\operatorname{PLT}\left(10^{9} / \mathrm{L}\right)$ & $266(112)$ & $249(102)$ & 0.050 \\
\hline MPV, fl & $8.5(1.5)$ & 8.4 & 0.651 \\
\hline PCT & $0.23(0.07)$ & $0.21(0.09)$ & 0.056 \\
\hline PDW & $16.6(0.4)$ & $16.5(1.6)$ & 0.604 \\
\hline Hb (g/dl) & $13.8(2.7)$ & $12.7(2.8)$ & 0.008* \\
\hline Het (\%) & $41.1(7.4)$ & $37.9(7.8)$ & $0.014 *$ \\
\hline Hb to RDW & $0.92 \pm 0.18$ & $0.87 \pm 0.19$ & 0.092 \\
\hline LMR & $3.1(2.58)$ & $3.1(2.48)$ & 0.680 \\
\hline PLR & $142(106)$ & 147 (109) & 0.533 \\
\hline MPV to PLT & $0.031(0.015)$ & $0.033(0.017)$ & 0.127 \\
\hline NLR & $2.4(2.2)$ & $3.7(4.7)$ & $<0.001 *$ \\
\hline HERR score & $3.0(1.0)$ & $4.0(2.0)$ & $<0.001 *$ \\
\hline
\end{tabular}

Data are shown as median (interquartile range). or number or mean \pm standard deviation. $\mathrm{F}=$ female, $\mathrm{Hb}=$ hemoglobin, HbRDW = hemoglobin to red cell distribution width, Hct $=$ hematocrit, HERR $=$ hemogram-related risk, $\mathrm{LMR}=$ lymphocyte to monocyte ratio, $\mathrm{M}=$ male, $\mathrm{MPV}=$ mean platelet volume, $\mathrm{NLR}=$ neutrophile to lymphocte ratio, $\mathrm{RCC}=$ renal cell carcinoma, $\mathrm{PLR}=$ platelet to lymphocte ratio, $\mathrm{PLT}=$ platelet count, $\mathrm{RDW}=$ red blood cell distribution width, $\mathrm{ROC}=$ receiver operating characteristic, $\mathrm{SE}=$ standard error, $\mathrm{WBC}=$ white blood cell, ${ }^{*} p<0.05$ 
Table 3. ROC curve analysis, and cut-off values for prediction of RCC from hemogram

\begin{tabular}{lccccc}
\hline Parameter & AUC & SE & $p$ value & $\begin{array}{c}\text { 95\% confidence } \\
\text { interval }\end{array}$ & Cut-off value \\
\hline MPV to PLT & 0.564 & 0.042 & 0.129 & $0.481-0.647$ & 0.029 \\
Hb to RDW & 0.582 & 0.041 & 0.053 & $0.501-0.663$ & 0.86 \\
PLR & 0.526 & 0.041 & 0.533 & $0.447-0.606$ & 120 \\
LMR & 0.508 & 0.041 & 0.842 & $0.429-0.588$ & 4.2 \\
NLR & 0.657 & 0.035 & $0.000 *$ & $0.588-0.727$ & 2.47 \\
\hline
\end{tabular}

$\mathrm{AUC}=$ Area under curve, $\mathrm{Hb}=$ hemoglobin, $\mathrm{LMR}=$ lymphocyte to monocyte ratio, $\mathrm{MPV}=$ mean platelet volume, $\mathrm{NLR}=$ neutrophile to lymphocte ratio, $\mathrm{RCC}=$ renal cell carcinoma, PLR $=$ platelet to lymphocte ratio, $\mathrm{PLT}=$ platelet count, $\mathrm{RDW}=$ red blood cell distribution width, $\mathrm{ROC}=$ receiver operating characteristic, $\mathrm{SE}=$ standard error

the RCC and benign-mass patients (Table 2). The ROC analysis (Table 3 ) showed the ideal cut-off value for the NLR was 2.47 (area under curve [AOC]: $0.657)$, and 4.2 was the ideal cut-off value for the LMR (AUC: 0.508). The ROC analysis also showed that the discriminatory power for NLR was greater than any other individual parameter.

As mentioned above, the continuously coded parameters were analyzed as categorical variables that were subsequently used to calculate the HERR score. Using the $\mathrm{Hb}$ to $\mathrm{RDW}$ ratio alone $(\leq 0.86)$ for diagnosing RCC, we obtained a specificity of 67 , meaning that it correctly identified $67 \%$ of those who did not have RCC. Patients with benign masses showed a median HERR score of 3 (IQR: 2.7) while the RCC median HERR score was 4 (2.0) $(p<0.001$; Figure 2). We found the cut off value of the HERR Score as 3 . The best value of the sensitivity and specificity of the parameters related to the hemogram is the cut off value. We calculated the cut off value as 3 , in this sampling. Patients with a high HERR score ( $\geq 3$ ) were more likely to have RCC. Using the $\geq 3$

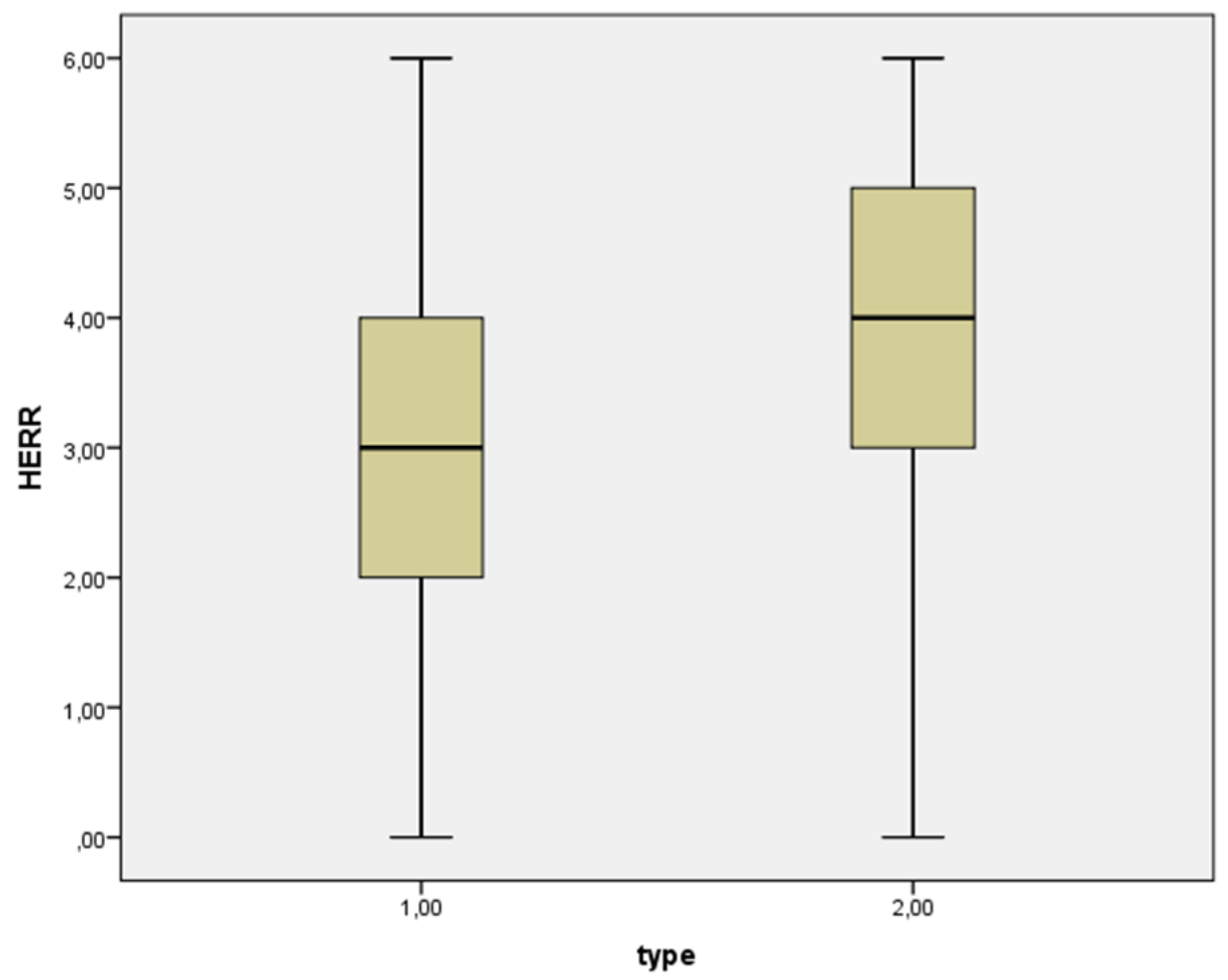

Figure 2. Hemogram related risk score. The bar graphs show blood HERR scores in benign (type 1) and RCC (type 2) patients. 
Table 4. Diagnostic power of hemogram-related indices

\begin{tabular}{|c|c|c|c|c|c|c|}
\hline Parameter & $\begin{array}{c}\text { SE (\%) } \\
(95 \% \mathrm{CI})\end{array}$ & $\begin{array}{c}\text { SP (\%) } \\
(95 \% \text { CI) }\end{array}$ & $\begin{array}{l}\text { PPV (\%) } \\
(95 \% \text { CI })\end{array}$ & $\begin{array}{l}\text { NPV (\%) } \\
(95 \% \text { CI) }\end{array}$ & $\begin{array}{c}\text { LR+* } \\
(95 \% \mathrm{CI})\end{array}$ & $\begin{array}{c}\text { LR-* } \\
(95 \% \text { CI })\end{array}$ \\
\hline Tumor size $(\geq 4 \mathrm{~cm})$ & $\begin{array}{c}37 \\
(31-43)\end{array}$ & $\begin{array}{c}75 \\
(61-85)\end{array}$ & $\begin{array}{c}88 \\
(80-93)\end{array}$ & $\begin{array}{c}20 \\
(15-26)\end{array}$ & $\begin{array}{c}7.3 \\
(4.4-12.0)\end{array}$ & $\begin{array}{c}4.0 \\
(3.6-4.5)\end{array}$ \\
\hline MPV to PLT ( $\geq$ cut-off) & $\begin{array}{c}82 \\
(77-87)\end{array}$ & $\begin{array}{c}26 \\
(16-40)\end{array}$ & $\begin{array}{c}84 \\
(79-88)\end{array}$ & $\begin{array}{c}24 \\
(14-37)\end{array}$ & $\begin{array}{c}5.5 \\
(4.1-7.3)\end{array}$ & $\begin{array}{c}3.1 \\
(2.4-3.9)\end{array}$ \\
\hline Hb to RDW (< cut-off) & $\begin{array}{c}47 \\
(41-53)\end{array}$ & $\begin{array}{c}67 \\
(53-79)\end{array}$ & $\begin{array}{c}88 \\
(81-92)\end{array}$ & $\begin{array}{c}12 \\
(15-27)\end{array}$ & $\begin{array}{c}7.2 \\
(4.7-11.2)\end{array}$ & $\begin{array}{c}3.7 \\
(3.3-4.2)\end{array}$ \\
\hline PLR ( $\geq$ cut-off) & $\begin{array}{c}69 \\
(63-74)\end{array}$ & $\begin{array}{c}44 \\
(31-58)\end{array}$ & $\begin{array}{c}86 \\
(80-90)\end{array}$ & $\begin{array}{c}22 \\
(15-31)\end{array}$ & $\begin{array}{c}6.0 \\
(4.3-8.4)\end{array}$ & $\begin{array}{c}3.4 \\
(2.9-4.0)\end{array}$ \\
\hline LMR (< cut-off) & $\begin{array}{c}70 \\
(65-75)\end{array}$ & $\begin{array}{c}29 \\
(24-34)\end{array}$ & $\begin{array}{c}83 \\
(77-87)\end{array}$ & $\begin{array}{c}17 \\
(12-22)\end{array}$ & $\begin{array}{c}4.8 \\
(3.5-6.4)\end{array}$ & $\begin{array}{c}5.1 \\
(4.2-6.2)\end{array}$ \\
\hline NLR ( $\geq$ cut-off) & $\begin{array}{c}68 \\
(62-73)\end{array}$ & $\begin{array}{c}51 \\
(38-65)\end{array}$ & $\begin{array}{c}87 \\
(82-91)\end{array}$ & $\begin{array}{c}25 \\
(17-34)\end{array}$ & $\begin{array}{c}6.9 \\
(4.8-9.8)\end{array}$ & $\begin{array}{c}3.0 \\
(2.5-3.5)\end{array}$ \\
\hline HERR score $(\geq 3)$ & $\begin{array}{c}78 \\
(73-83) \\
\end{array}$ & $\begin{array}{c}69 \\
(56-80) \\
\end{array}$ & $\begin{array}{c}91 \\
(87-94) \\
\end{array}$ & $\begin{array}{c}44 \\
(34-54) \\
\end{array}$ & $\begin{array}{c}10.8 \\
(7.0-16.4) \\
\end{array}$ & $\begin{array}{c}1.2 \\
(1.0-1.5) \\
\end{array}$ \\
\hline
\end{tabular}

$\mathrm{Hb}=$ hemoglobin, HERR $=$ hemogram-related risk, LMR = lymphocyte to monocyte ratio, LR $+=$ positive likelihood ratio, $\mathrm{LR}-$ = negative likelihood ratio, $\mathrm{MPV}=$ mean platelet volume, $\mathrm{MPV}$ to $\mathrm{PLT}=$ mean platelet volume to platelet ratio, $\mathrm{PPV}=$ positive predictive value, $\mathrm{NLR}=$ neutrophile to lymphocte ratio, $\mathrm{NPV}=$ negative predictive value, PLR = platelet to lymphocte ratio, PLT = platelet count, RDW = red blood cell distribution width, $\mathrm{SE}=$ sensitivity, $\mathrm{SP}=$ specificity, ${ }^{*}$ weighted by prevelance

HERR score cut-off, we obtained a specificity of $69 \%$ (95\% confidence interval $[\mathrm{CI}]: 56-80)$, meaning that it correctly identified of $69 \%$ of those who did not have a malign tumor. We obtained a high $\mathrm{LR}+$ ratio (10.8) for the HERR scores that are in the best indicator range for ruling the diagnosis as RCC, and a PPV of 91 (87-94) within this category of HERR score (Table 4).

\section{DISCUSSION}

We hypothesized that there might be different degrees of circulating blood cell indices in RCC as compared to benign masses, and we explored the possibility that these indices could distinguish RCC from other benign renal masses. To provide comprehensive information for diagnosing RCC patients, we evaluated the NLR, PLR, LMR, and $\mathrm{Hb}$ to RDW ratios, and integrated them into a reliable scoring system that could be performed from a routine blood count to detect samples that could indicate RCC. While calculating the HERR score, platelet, monocyte, lymphocyte, neutrophil, and erythrocyte levels were evaluated simultaneously. Thus, a high HERR score might reflect alterations that favor cancer initiation and progression.

Our findings demonstrated that combining hemogram parameters into an integrated HERR score to discriminate among the kidney masses preoperatively offered a low rate of false positives and an adequate $\mathrm{LR}+$ level as compared to using these values separately. In the current study, we found that none of the evaluated hematological indices mentioned in the literature were significantly correlated with a diagnosis of RCC and that the HERR score was significantly better than any of these parameters.

Additionally, the preoperative NLR score had powerful diagnostic abilities compared to the other hematologic indices, and it showed the highest diagnostic specificity and sensitivity. The NLR combined the measurements of the increased protumor activity of neutrophils with those of the reduced antitumor immune response by lymphocytes into a single value $[7,8]$. We found that the NLR levels were higher in the RCC group, which can reflect the status of the tumor microenvironment. Similarly to our study, a number of researchers have demonstrated that the preoperative NLR value was significantly higher in malignant renal masses compared to benign ones [8, 
$13,14]$.

Platelets have been shown to facilitate tumor progression by contributing to the metastatic cascade, regulating tumor cell invasion, and playing a role in angiogenesis [15]. A meta-analysis reported by Wang et al. [9] reported that an elevated PLR and reduced LMR were associated with poorer overall survival in RCC patients. Monocytes can be recruited in tumor tissues and exert pre-tumoral actions, meaning that a decreased LMR could be associated with the less favorable prognosis that was observed in RCC [9].

Previously, the MPV/PLT ratio as a predictor of cancer gave inconsistent results $[16,17]$. In our study, this ratio for RCC patients and the benign group was comparable. We did not find any previous reports assessing the MPV/PLT ratio for patients with RCC.

$\mathrm{The} \mathrm{Hb}$ to RDW levels were non-significantly reduced in RCC compared to benign cases. Recently, a low $\mathrm{Hb} / \mathrm{RDW}$ ratio was reported to be significantly associated with poor clinical outcomes and a greater risk of death in esophageal squamous cell carcinoma patients [10]. Because both $\mathrm{HB}$ and RDW are influenced by various non-cancer-related conditions, the $\mathrm{Hb} / \mathrm{RDW}$ could therefore theoretically reflect generalized health information, such as the nutrition status, inflammatory status, and immune function.

All of the blood parameters measured are available in routine blood tests, are easy to perform by the vast majority of automated analyzers, and do not increase the cost of diagnosis. The HERR score in combination with a summary of clinical symptoms might alert physicians to the early detection of RCC. The goal of a reliable screening test is to get as close as possible to zero false negative results with a minimal percentage of false positive results. Although hematologic indices are easy to measure, their utility might be affected by several factors that could lead to different cut-off points from those already mentioned in every hospital [18].

\section{Limitations}

The limitations of the present study include its retrospective nature and relatively small number of patients located at only one center. Due to biological differences between distinct patient cohorts, further studies in different cohorts with external validation are needed.

\section{CONCLUSION}

In conclusion, our study, despite being a preliminary validation, is the first to evaluate hemogram-related parameters for preoperatively discriminating between RCC and benign renal masses. The HERR score developed herein is a potential diagnostic biomarker that aids in this discrimination.

\section{Conflict of interest}

The authors disclosed no conflict of interest during the preparation or publication of this manuscript.

\section{Financing}

The authors disclosed that they did not receive any grant during conduction or writing of this study.

\section{REFERENCES}

[1] Siegel RL, Miller KD, Jemal A. Cancer statistics, 2016. CA. Cancer J Clin 66 2016;66:7-30.

[2] Tahbaz R, Schmid M, Merseburger AS. Prevention of kidney cancer incidence and recurrence: lifestyle, medication and nutrition. Curr Opin Urol 2018;28:62-79.

[3] Acar C, Sözen S, Üre I, Batur AF, Gürocak S, Küpeli B. Nephronsparing treatments in small renal tumors: surgical and ablative procedures. Turk J Urol 2009;35:87-95.

[4] Özen H, Colowick A, Freiha FS. Incidentally discovered solid renal masses: what are they? Br J Urol 1993;72:274-6.

[5] Kay FU, Pedrosa I. Imaging of solid renal masses. Radiol Clin North Am 2017;55:243-58.

[6] Lane BR, Babineau D, Kattan MW, Novick AC, Gill IS, Zhou M, et al. A preoperative prognostic nomogram for solid enhancing renal tumors $7 \mathrm{~cm}$ or less amenable to partial nephrectomy. J Urol 2007; 178:429-34.

[7] Grivennikov SI, Greten FR, Karin M. Immunity, inflammation, and cancer. Cell 2010;140:883-99.

[8] Hu K, Lou L, Ye J, Zhang S. Prognostic role of theneutrophillymphocyteratio in renal cell carcinoma: a meta-analysis. BMJ Open 2015;5:e06404.

[9] Wang X, Su S, Guo Y. The clinical use of the platelet to lymphocyte ratio and lymphocyte to monocyte ratio as prognostic factors in renal cell carcinoma: a systematic review and meta-analysis. Oncotarget 2017;8:84506-14.

[10] Sun P, Zhang F, Chen C, Bi X, Yang H, An X, et al. The ratio of hemoglobin to red cell distribution width as a novel prognostic parameter in esophageal squamous cell carcinoma: a retrospective study from southern China. Oncotarget 2016;5:42650-60.

[11] Prokopowicz G, Życzkowski M, Nowakowski K, Bogacki R, Bryniarski P, Paradysz A. Basic parameters of blood count as prognostic factors for renal cell carcinoma. Biomed Res Int 2016;2016:8687575. [12] Golovastova MO, Korolev DO, Tsoy LV, Varshavsky VA, Xu WH, Vinarov AZ, et al. Biomarkers of renal tumors: the current state and clinical perspectives. Curr Urol Rep 2017;18:3.

[13] Gorgel SN, Ozer K, Kose O, Dindar AS. Can preoperative 
neutrophil lymphocyte ratio predict malignancy in patients undergoing partial nephrectomy because of renal mass? Int Braz J Urol 2017;43. doi: 10.1590/S1677-5538.IBJU.2017.0225.

[14] Viers BR, Thompson RH, Lohse CM, Cheville JC, Leibovich BC, Boorjian SA, et al. Pre-treatment neutrophil-to-lymphocyte ratio predicts tumor pathology in newly diagnosed renaltumors. World J Urol 2016;34:1693-9.

[15] Stegner D, Dutting S, Nieswandt B. Mechanistic explanation for platelet contribution to cancer metastasis. Thromb Res 2014;2:149-57. [16] Cho SY, Yang JJ, You E, Kim BH, Shim J, Lee HJ, et al. Mean platelet volume/platelet count ratio in hepatocellular carcinoma. Platelets 2013;24:375-7.

[17] Inagaki N, Kibata K, Tamaki T, Shimizu T, Nomura S. Prognostic impact of the mean platelet volume/platelet count ratio in terms of survival in advanced non-small cell lung cancer. Lung Cancer 2014;83:97-101.

[18] Budak YU, Polat M, Huysal K. The use of platelet indices, plateletcrit, mean platelet volume and platelet distribution width in emergency non-traumatic abdominal surgery: a systematic review. Biochem Med (Zagreb) 2016;26:178-93. 